This item was submitted to Loughborough's Research Repository by the author.

Items in Figshare are protected by copyright, with all rights reserved, unless otherwise indicated.

\title{
Total synthesis of the marine-derived cyclic depsipeptide alternaramide
}

\section{PLEASE CITE THE PUBLISHED VERSION}

http://dx.doi.org/10.1055/s-0030-1259915

\section{PUBLISHER}

(c) Georg Thieme Verlag

\section{VERSION}

AM (Accepted Manuscript)

\section{LICENCE}

CC BY-NC-ND 4.0

\section{REPOSITORY RECORD}

Horton, Alexandra E., Oliver S. May, Mark R.J. Elsegood, and Marc C. Kimber. 2019. "Total Synthesis of the Marine-derived Cyclic Depsipeptide Alternaramide”. figshare. https://hdl.handle.net/2134/13937. 
This item was submitted to Loughborough's Institutional Repository (https://dspace.lboro.ac.uk/) by the author and is made available under the following Creative Commons Licence conditions.

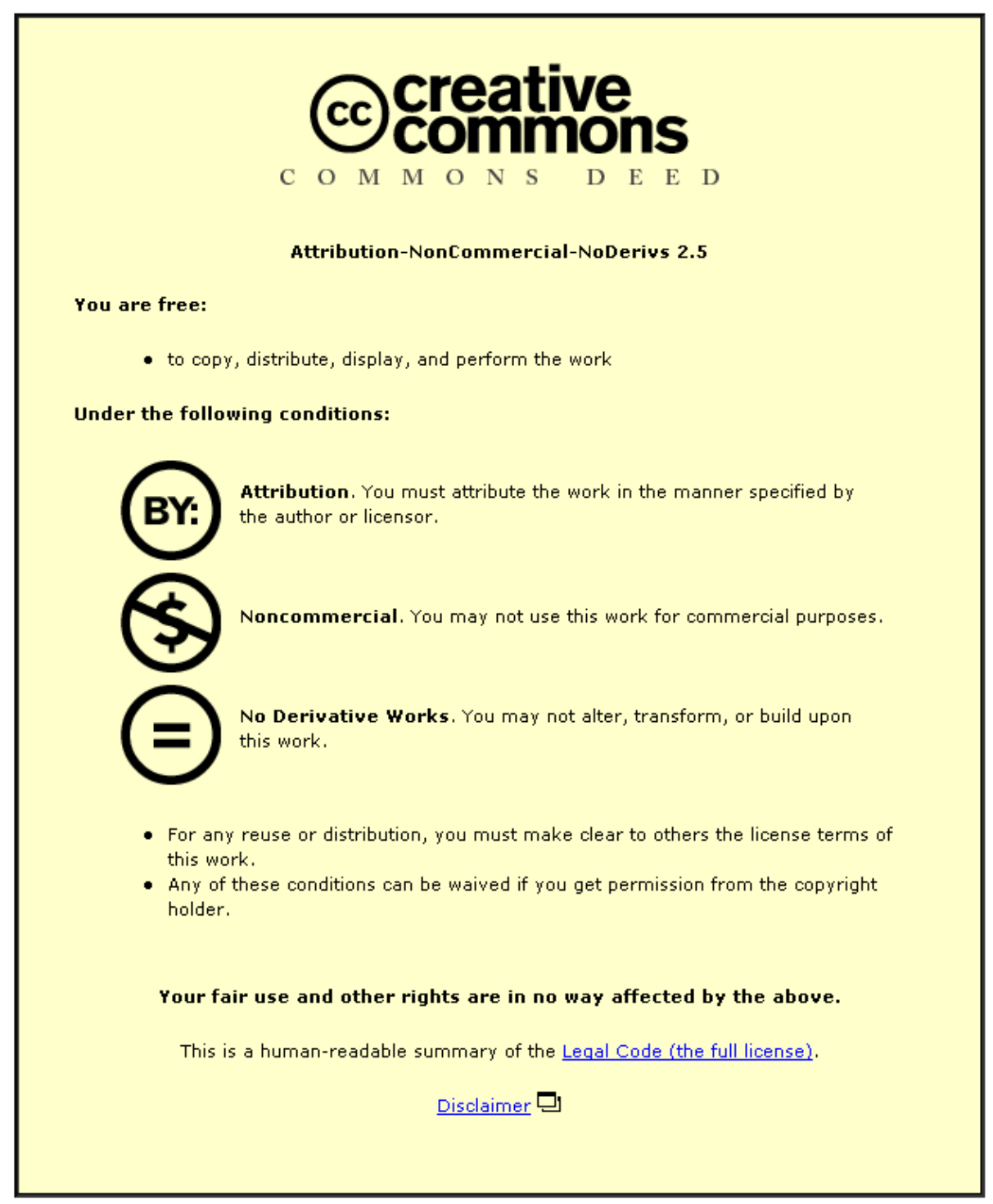

For the full text of this licence, please go to: http://creativecommons.org/licenses/by-nc-nd/2.5/ 


\title{
The total synthesis of the marine-derived cyclic depsipeptide alternaramide
}

\author{
Alexandra E. Horton, Oliver S. May, Mark R. J. Elsegood and Marc C. Kimber* \\ The Department of Chemistry, Loughborough University, Loughborough, Leicester, LE11 3TU, UK \\ Fax: +44(0)-150-922-3925 \\ E-mail: M.C.Kimber@lboro.ac.uk \\ Received: The date will be inserted once the manuscript is accepted.
}

Abstract: The first synthesis of the marine fungus derived natural product alternaramide is described using solution phase coupling protocols and via a macrolactonization and macrolactamization route. The structure of alternaramide was confirmed and was supported by single crystal X-ray analysis which exhibited three similar molecules in the asymmetric unit, each with trans-annular hydrogen bonds.

Key words: Natural product, depsipeptide, peptide coupling, macrolactonization, macrolactamization.

Alternaramide 1 (Figure 1) is a cyclic depsipeptide ${ }^{1}$ isolated from the marine-derived fungus Alternaria $s p$. SF5016 by $\mathrm{Oh}$ and co-workers in 2009 . $^{2}$

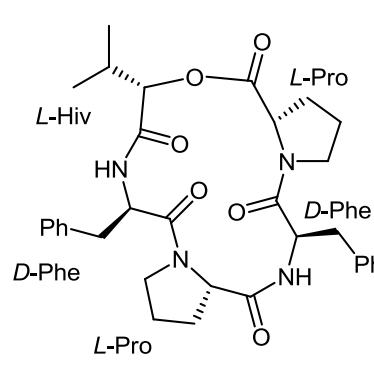

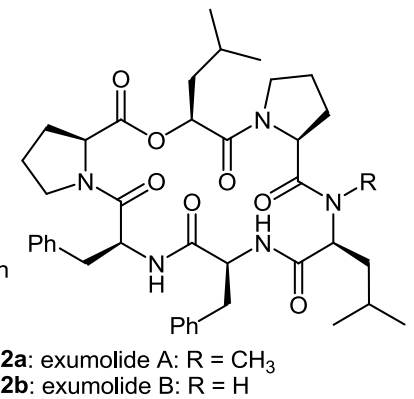<smiles>CC(C)CC1NC(=O)C(CC(C)C)NC(=O)C(C(C)C)NC(=O)C(Cc2ccccc2)C(CC(C)C)NC(=O)C(CC(C)C)NC1=O</smiles><smiles>CC(C)CC1NC(=O)C(Cc2ccccc2)NC(=O)C(Cc2ccccc2)NC(=O)C(CC(C)C)OC(=O)C(Cc2ccccc2)NC(=O)C(CC(C)C)NC1=O</smiles>

Figure 1. The reported ${ }^{2}$ structure of alternaramide 1 and related marine-derived depsipeptide natural products.

The structure of $\mathbf{1}$ is related to several depsipeptides isolated from marine-derived fungi, such as the exumolides $\mathbf{2}$ from Scytalidium sp., ${ }^{3}$ sansalvamide A $\mathbf{3}$ from Fusarium sp., ${ }^{4}$ and zygosporamide 4 from Zygosporium masonii., ${ }^{5}$ and was confirmed using a mixture of ${ }^{1} \mathrm{H} /{ }^{13} \mathrm{C}$ NMR, degradation and Mosher's ester derivitization studies. This showed that the depsipeptide contained 4 amino acid residues (two $L$ Pro and two $D$-Phe) and one hydroxy acid residue ( $L$-Hiv) linked by 4 amide and 1 ester linkage. While all these peptides possess hydrophobic amino acid residues, the presence of the $D$-Phe residue in $\mathbf{1}$ is unusual since the only other depsipeptide in this group to have $D$-amino acid residues is zygosporamide 4 (Figure 1). Additionally, the importance of a $D$-amino acid in analogues of sansalvamide $\mathrm{A}$, in relation to anticancer activity, has been determined by
McGuire and McAlpine via SAR studies. ${ }^{6-10}$ Total syntheses of $\mathbf{3}^{11}$ and $\mathbf{4}^{12}$ have been described previously, however the total synthesis of $\mathbf{1}$ has yet to be reported. Therefore, in this letter, we would like to report a short, efficient, solution phase total synthesis of $\mathbf{1}$, confirmation of its reported structure and a single crystal X-ray analysis.

The retrosynthesis of $\mathbf{1}$ is shown in Scheme 1 . Alternaramide $\mathbf{1}$ can be obtained via either a macrolactonization or a macrolactamization event on the precursor linear peptides $\mathbf{5}$ and $\mathbf{6}$, respectively. While there are examples of the use macrolactonization in the synthesis of depsipeptides, ${ }^{13-16}$ such a cyclisation performed on $\mathbf{5}$ may be problematic. However, due to the short synthetic sequence to give the precursor $\mathbf{5}$ we thought this would be a viable route to explore, alongside the macrolactamization approach. The synthesis of both precursor linear peptides 5 and 6 would be from the key tetrapeptide 7 using standard peptide coupling protocols. The tetrapeptide 7 would be obtained from $L$-Pro, $D$-Phe, and $L$-Hiv, which can be obtained from $L$-Val via hydrolytic diazotization.

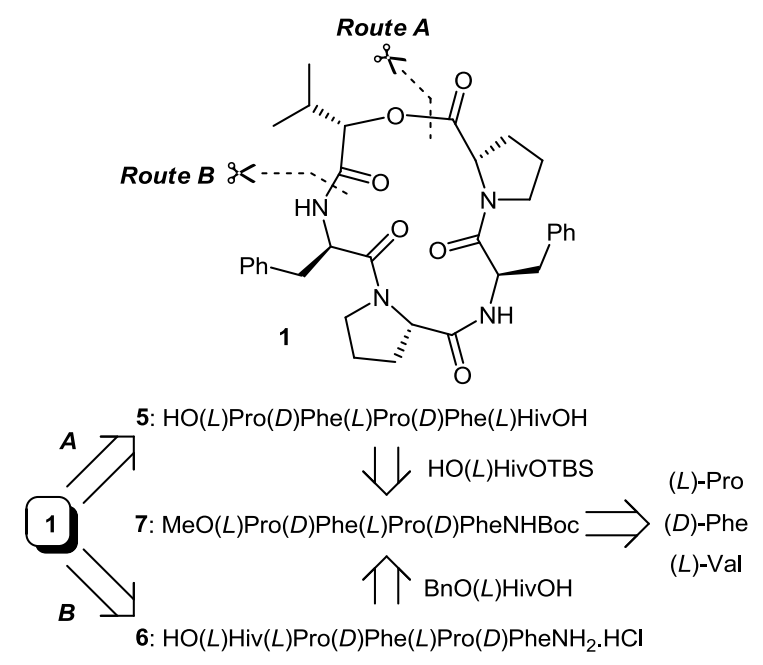

Scheme 1. Retrosynthetic analysis of alternaramide $\mathbf{1 .}$

Coupling of proline methyl ester 8 with $\mathrm{HO}(D) \mathrm{PheHNBoc}$ 9 was initially performed using two coupling methods, either EDC or formation of the mixed anhydride which gave the diamino acid 12 in a good yield of $87 \%$ and $86 \%$, respectively (Scheme 2). PyBOP ${ }^{\circledR}$ was also employed but yields using this coupling reagent were moderate to poor. The TBS protected $\alpha$-hydroxy acid $\mathbf{1 3}$ was obtained in 3steps in $65 \%$ yield via the hydrolytic diazotization of $L$-Val $\mathbf{1 0}$ followed by TBS protection. ${ }^{17}$ 


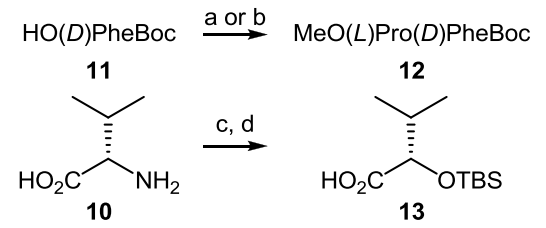

Scheme 2. (a) $\mathrm{EDC}, \mathrm{MeO}(L) \mathrm{ProNH}_{2} \cdot \mathrm{HCl}, \mathrm{CH}_{2} \mathrm{Cl}_{2},{ }^{i} \mathrm{Pr}_{2} \mathrm{NEt}$, [87\%]; (b) $\mathrm{EtO}_{2} \mathrm{CCl}, \mathrm{NMM}, \mathrm{CH}_{2} \mathrm{Cl}_{2}, 0^{\circ} \mathrm{C}$ then, $\mathrm{MeO}(L) \operatorname{ProNH}_{2} \cdot \mathrm{HCl}$, [86\%]; (c) $\mathrm{NaNO}_{2}, \mathrm{H}_{2} \mathrm{SO}_{4}$ (d) (i) $\mathrm{TBSCl}, \mathrm{DMF}$, imidazole (ii) $\mathrm{K}_{2} \mathrm{CO}_{3}, \mathrm{MeOH}, \mathrm{H}_{2} \mathrm{O}$ [65\% over 2-steps].

The key tetrapeptide 7 was obtained in 2-steps from 12, by Boc deprotection of $\mathbf{1 2}$ with $4 \mathrm{M} \mathrm{HCl}$ in 1,4-dioxine to give $\mathbf{1 4}$, and the hydrolysis of $\mathbf{1 2}$ to the free acid using $\mathrm{LiOH}$ to give 15, both of which were used without further purification. The salt 14 and free acid 15 were then coupled under EDC conditions to give the key tetra-aminoacid $\mathbf{7}$ in $52 \%$ yield over the 2 -steps (Scheme 3 ).

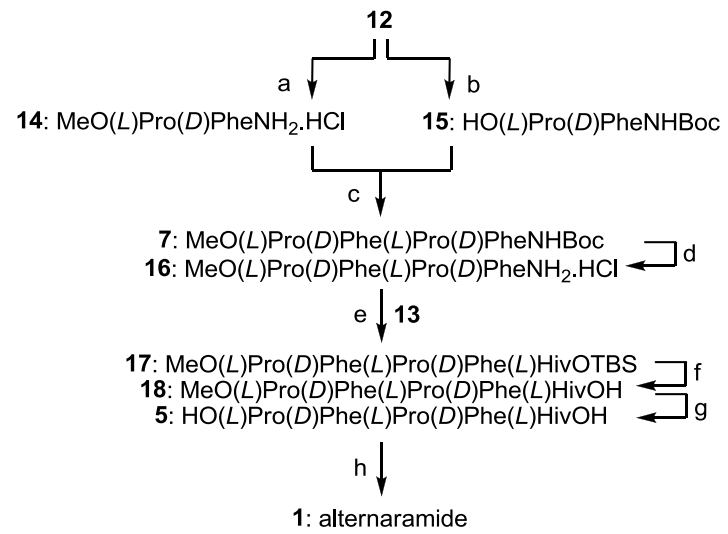

Scheme 3. (a) $4 \mathrm{M} \mathrm{HCl}$ in 1,4-dioxane; (b) $\mathrm{LiOH}, \mathrm{THF}, \mathrm{MeOH}$, $\mathrm{H}_{2} \mathrm{O}$; (c) EDC, $\mathrm{CH}_{2} \mathrm{Cl}_{2},{ }^{i} \mathrm{Pr}_{2} \mathrm{NEt}_{2}$ [52\% over 2-steps]; (d) $4 \mathrm{M} \mathrm{HCl}$ in 1,4-dioxane; (e) $\mathrm{PyBOP}^{\circledR}, \mathrm{CH}_{2} \mathrm{Cl}_{2},{ }^{i} \mathrm{Pr}_{2} \mathrm{NEt}$ [71\% over 2-steps]; (f) TBAF, THF; (g) $\mathrm{LiOH}, \mathrm{THF}, \mathrm{MeOH}, \mathrm{H}_{2} \mathrm{O}$; (h) $\mathrm{PyBOP}^{\circledR}$, $\mathrm{CH}_{2} \mathrm{Cl}_{2},{ }^{i} \mathrm{Pr}_{2} \mathrm{NEt}$ [5\% over 3-steps].

Subsequently, 7 was Boc deprotected and the resultant salt $\mathbf{1 6}$ then coupled with the $\mathbf{1 3}$ using $\mathrm{PyBOP}^{\circledR}$ giving $\mathbf{1 7}$ in a good yield of $71 \%$ over 2 -steps. The free alcohol and acid on 17 were then sequentially revealed by deprotection using TBAF and followed by treatment with excess aqueous $\mathrm{LiOH}$. Attempted final ring closure of $\mathbf{5}$ was then trialed with a number of coupling reagents including $\mathrm{PyBOP}^{\circledR}$, DCC, EDC and 1,3,5-trichlorobenzoyl chloride. ${ }^{18}$ The only successful conditions were using excess $\mathrm{PyBOP}^{\circledR}$ and diisopropylethylamine which provided alternaramide $\mathbf{1}$ in a poor isolated yield of only $5 \%$ over the 3 -steps, presumably due to the low nucleophilicity of the hydroxyl group. Consequently, route B (Scheme 1) with the final ring closure via a macrolactamization was pursued.

The requisite substrate for the macrolactamization was synthesized in 4-steps from the key tetrapeptide 7 (Scheme 4). A protecting group switch on the $L$-Hiv fragment was achieved in 2-steps to give the benzyl ester protected $L$-Hiv fragment 19 in 52\% yield. This was then successfully coupled to the saponified tetrapeptide $\mathbf{2 0}$ in $\mathbf{7 3 \%}$ yield over the 2-steps. Finally, the free acid and amine on $\mathbf{2 0}$ were revealed by sequential hydrogenolysis followed by acid mediated Boc deprotection. The macrolactamization of $\mathbf{6}$ was then achieved using excess PyBOP $^{\circledR}$ with diisopropyl amine and catalytic DMAP, giving alternaramide 1 in $48 \%$ yield over the 3 -steps. ${ }^{19}$

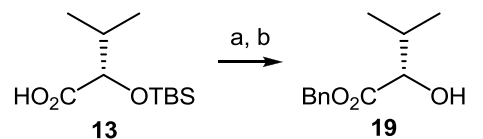

7: $\mathrm{MeO}(L) \operatorname{Pro}(D) \operatorname{Phe}(L) \operatorname{Pro}(D)$ PheNHBoc 20: $\mathrm{HO}(L)$ Pro( $D)$ Phe $(L)$ Pro( $(D)$ PheNHBoc $\square c$

$$
d \downarrow 19
$$

21: $\mathrm{BnO}(L) \operatorname{Hiv}(L) \operatorname{Pro}(D) \operatorname{Phe}(L) \operatorname{Pro}(D) \mathrm{PheNHBoc} \square \mathrm{e}$

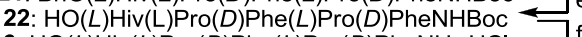
6: $\mathrm{HO}(L) \mathrm{Hiv}(L) \operatorname{Pro}(D) \operatorname{Phe}(L) \operatorname{Pro}(D) \mathrm{PheNH}_{2} . \mathrm{HCl}$

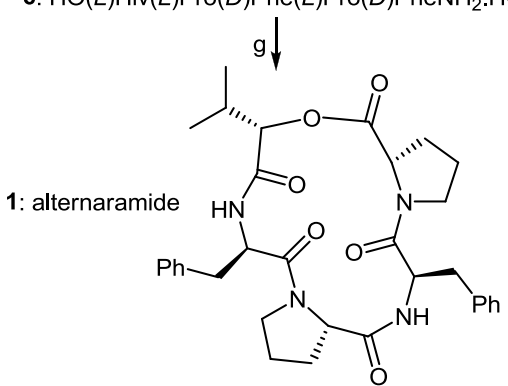

Scheme 4. (a) $\mathrm{BnC}(\mathrm{O}) \mathrm{Cl}, \mathrm{CH}_{2} \mathrm{Cl}_{2}, \mathrm{Et}_{3} \mathrm{~N}$, DMAP; (b) TBAF, THF [52\% over 2-steps]; (c) $\mathrm{LiOH}, \mathrm{MeOH}, \mathrm{THF}, \mathrm{H}_{2} \mathrm{O}$; (d) $\mathrm{PyBOP}^{\circledR}$, $\mathrm{CH}_{2} \mathrm{Cl}_{2},{ }^{i} \mathrm{Pr}_{2} \mathrm{NEt}$ [73\% over 2-steps]; (e) Pd-C (10\%), $\mathrm{H}_{2}$, MeOH; (f) $4 \mathrm{M} \mathrm{HCl}$ in 1,4-dioxane; (g) PyBOP ${ }^{\circledR}, \mathrm{CH}_{2} \mathrm{Cl}_{2},{ }^{i} \mathrm{Pr}_{2} \mathrm{Net}$, DMAP (cat.) [48\% over 3 -steps].

The spectroscopic data for our synthetic alternaramide sample agreed well with that reported by $\mathrm{Oh}$ and coworkers and copies of the relevant ${ }^{1} \mathrm{H}$ and ${ }^{13} \mathrm{C}$ NMR spectra are contained within the supplementary information. ${ }^{20,21}$ Crystals suitable for single crystal X-ray analysis were obtained and Figure 2 shows the molecular depiction of one of three similar molecules of (-)-1 in the asymmetric unit. ${ }^{22}$ Despite the use of Mo-K $\alpha$ radiation for this light atom structure, determination of the absolute structure parameter $^{23}$ via Parson's Q-value method ${ }^{24}$ led to a value that supported the proposed absolute structure for the molecule. The molecular conformation is stabilised by trans-annular hydrogen bonds.

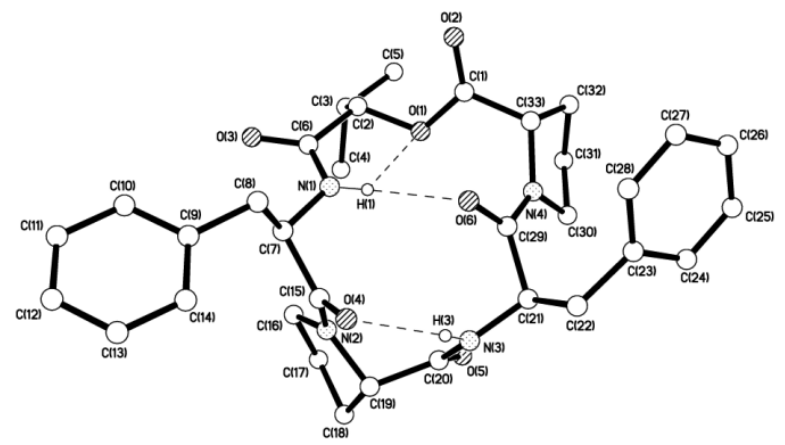

Figure 2. Crystal structure representation of alternaramide (-)-1. ${ }^{22}$

In conclusion, we have reported the first total synthesis of alternaramide (-)-1 using standard solution phase peptide coupling protocols. Two synthetic pathways have been investigated using a macrolactonization and a macrolactamization approach giving alternaramide in a best overall yield of $11 \%$ in 8 -steps. Finally, the structure of synthetic alternaramide (-)-1 was confirmed by single crystal X-ray analysis. 
Supporting Information for this article includes the ${ }^{1} \mathrm{H}$ and ${ }^{13} \mathrm{C}$ NMR spectra of synthetic alternaramide (-)-1 and crystallographic data for (-)-1.

\section{Acknowledgments}

The authors thank the Department of Chemistry at Loughborough University for financial support.

\section{References and Notes}

(1) (a) Ballard, C. E.; Yu, H.; Wang, B. Curr. Med. Chem. 2002, 9, 471. (b) Li, W.; Schlecker, A.; Ma, D. Chem. Commun. 2010, 46, 5403.

(2) Kim, M.-Y.; Sohn, J. H.; Ahn, J. S.; Oh, H. J. Nat. Prod. 2009, 72, 2065.

(3) Jenkins, K. M.; Renner, M. K.; Jensen, P. R.; Fenical, W. Tetrahedron Lett. 1998, 39, 2463.

(4) Belofsky, G. N.; Jensen, P. R.; Fenical, W. Tetrahedron Lett. 1999, 40, 2913.

(5) Oh, D.-C.; Jensen, P. R.; Fenical, W. Tetrahedron Lett. 2006, $47,8625$.

(6) Alexander, L. D.; Sellers, R. P.; Davis, M. R.; Ardi, V. C.; Johnson, V. A.; Vasko, R. C.; McAlpine, S. R. J. Med. Chem. 2009, 52, 7927.

(7) Carroll, C. L.; Johnston, J. V. C.; Kekec, A.; Brown, J. D.; Parry, E.; Cajica, J.; Medina, I.; Cook, K. M.; Corral, R.; Pan, P.-S.; McAlpine, S. R. Org. Lett. 2005, 7, 3481.

(8) Otrubova, K.; Styers, T. J.; Pan, P.-S.; Rodriguez, R.; McGuire, K. L.; McAlpine, S. R. Chem. Commun. 2006, 1033.

(9) Styers, T. J.; Kekec, A.; Rodriguez, R.; Brown, J. D.; Cajica, J.; Pan, P.-S.; Parry, E.; Carroll, C. L.; Medina, I.; Corral, R.; Lapera, S.; Otrubova, K.; Pan, C.-M.; McGuireb, K. L.; McAlpinea, S. R. Bioorg. Med. Chem. 2006, 14, 5625.

(10) Vasko, R. C.; Rodriguez, R. A.; Cunningham, C. N.; Ardi, V. C.; Agard, D. A.; McAlpine, S. R. ACS Med. Chem. Lett. 2010, 1, 4.

(11) Lee, Y.; Silverman, R. B. Org. Lett. 2000, 2, 3743.

(12) Wanga, Y.; Zhang, F.; Zhang, Y.; Liu, J. O.; Ma, D. Bioorg. Med. Chem. Lett. 2008, 18, 4385.

(13) Cochrane, J. R.; McErlean, C. S. P.; Jolliffe, K. A. Org. Lett. 2010, 12, 3394.

(14) Doi, T.; Iijima, Y.; Shin-ya, K.; Ganesand, A.; Takahashia, T. Tetrahedron Lett. 2006, 47, 1177.

(15) Palomo, C.; Oiarbide, M.; Garcia, J. M.; Gonzalez, A.; Pazos, R.; Odriozola, J. M.; Banuelos, P.; Tello, M.; Linden, A. J. Org. Chem. 2004, 69, 4126.

(16) Sarabia, F.; Chammaa, S.; López-Herrera, F. J. Tetrahedron Lett. 2002, 43, 2961.

(17) Gonzalez, I.; Jou, G.; Caba, J. M.; Albericio, F.; LloydWilliams, P.; Giralt, E. J. Chem. Soc., Perkin Trans. 1 1996, 1427.

(18) Inanaga, J.; Hirata, K.; Saeki, H.; Katsuki, T.; Yamaguchi, M. Bull. Chem. Soc. Jpn. 1979, 52, 1989.

(19) Selected physical data for new compounds: $\mathrm{MeO}(L) \operatorname{Pro}(D)$ PheNHBoc, (-)-12. Colourless oil (3.05 g, $87 \%) ; \mathrm{R}_{f}\left(3: 1\right.$ ethyl acetate:petroleum ether) $0.65 ;[\alpha]_{\mathrm{D}}{ }^{19}-$ $5.5\left(\mathrm{c} 1, \mathrm{CHCl}_{3}\right) ; v_{\max }$ (solution, $\mathrm{CHCl}_{3}$ ) 3584, 3430, $2978,1746,1707,1645,1437,1171 \mathrm{~cm}^{-}{ }^{1} ; \delta_{\mathrm{H}}(400 \mathrm{MHz}$; $\left.\mathrm{CDCl}_{3}\right) 7.33-7.24(\mathrm{~m}, 5 \mathrm{H}), 5.55(\mathrm{~d}, J=8.8 \mathrm{~Hz}, 1 \mathrm{H}), 4.71$ - $4.69(\mathrm{~m}, 1 \mathrm{H}), 4.36(\mathrm{dd}, J=3.6,7.6 \mathrm{~Hz}, 1 \mathrm{H}), 3.75(\mathrm{~s}$, $3 \mathrm{H}), 3.62-3.60(\mathrm{~m}, 1 \mathrm{H}), 3.09-3.07(\mathrm{~m}, 1 \mathrm{H}), 3.02-2.98$ $(\mathrm{m}, 1 \mathrm{H}), 2.76-2.74(\mathrm{~m}, 1 \mathrm{H}), 1.98-1.90(\mathrm{~m}, 4 \mathrm{H}), 1.49(\mathrm{~s}$, $9 \mathrm{H}) ; \delta_{\mathrm{C}}\left(100 \mathrm{MHz} ; \mathrm{CDCl}_{3}\right) 172.21(\mathrm{C}), 170.22(\mathrm{C}), 154.91$ (C), $136.42(\mathrm{C}), 129.40(\mathrm{CH}), 128.28(\mathrm{CH}), 126.81(\mathrm{CH})$, $79.44(\mathrm{C}), 58.65(\mathrm{CH}), 53.53(\mathrm{CH}), 52.05\left(\mathrm{CH}_{3}\right), 46.66$
$\left(\mathrm{CH}_{2}\right), 40.07\left(\mathrm{CH}_{2}\right), 28.85\left(\mathrm{CH}_{2}\right), 28.25\left(\mathrm{CH}_{3}\right), 24.33$ $\left(\mathrm{CH}_{2}\right)$; HRMS $\mathrm{MNa}^{+}, \mathrm{C}_{20} \mathrm{H}_{28} \mathrm{~N}_{2} \mathrm{O}_{5}$, found 399.1885, requires 399.1896 .

$\mathrm{MeO}(L) \operatorname{Pro}(D) \operatorname{Phe}(L) \operatorname{Pro}(D) \operatorname{PheNHBoc}(-)-7$. White foam (0.97 g, 52\% over 2 -steps); $\mathrm{R}_{f}$ (3:1 ethyl acetate:petroleum ether) 0.38; $[\alpha]_{\mathrm{D}}^{21}-8.1$ (c $1, \mathrm{CHCl}_{3}$ ); $v_{\max }$ (solution, $\left.\mathrm{CHCl}_{3}\right)$ 3584, 3295, 2980, 1742, 1642, 1497, 1449, 1171 $\mathrm{cm}^{-1} ; \delta_{\mathrm{H}}\left(400 \mathrm{MHz} ; \mathrm{CDCl}_{3}\right) 7.25-7.17(\mathrm{~m}, 10 \mathrm{H}), 5.51(\mathrm{~d}$, $J=7.6 \mathrm{~Hz}, 1 \mathrm{H}), 4.86(\mathrm{dt}, J=6.4,8.4 \mathrm{~Hz}, 1 \mathrm{H}), 4.57-4.54$ (m, 1H), 4.36 (dd, $J=2.4,8.0 \mathrm{~Hz}, 1 \mathrm{H}), 4.27$ (dd, $J=4.0$, $8.4 \mathrm{~Hz}, 1 \mathrm{H}), 3.68(\mathrm{~s}, 3 \mathrm{H}), 3.53-3.49(\mathrm{~m}, 2 \mathrm{H}), 3.02-2.93$ (m, 4H), $2.77-2.75(\mathrm{~m}, 1 \mathrm{H}), 2.63-2.60(\mathrm{~m}, 1 \mathrm{H}), 2.46(\mathrm{~s}$, $1 \mathrm{H}), 2.03-1.98(\mathrm{~m}, 1 \mathrm{H}), 1.93-1.78(\mathrm{~m}, 4 \mathrm{H}), 1.61-1.50$ $(\mathrm{m}, 3 \mathrm{H}), 1.41(\mathrm{~s}, 9 \mathrm{H}) ; \delta_{\mathrm{C}}\left(100 \mathrm{MHz} ; \mathrm{CDCl}_{3}\right) 172.31(\mathrm{C})$, 171.08 (C), 170.43 (C), 169.53 (C), 155.21 (C), 136.62 (C), $129.54(\mathrm{CH}), 129.50(\mathrm{CH}), 129.33(\mathrm{C}), 128.46(\mathrm{CH})$, $128.34(\mathrm{CH}), 126.94(\mathrm{CH}), 126.84(\mathrm{CH}), 79.75(\mathrm{C}), 60.16$ $(\mathrm{CH}), 58.80(\mathrm{CH}), 53.92\left(\mathrm{CH}_{3}\right), 52.42(\mathrm{CH}), 52.22(\mathrm{CH})$, $46.84\left(\mathrm{CH}_{2}\right), 46.69\left(\mathrm{CH}_{2}\right), 39.63\left(\mathrm{CH}_{2}\right), 39.00\left(\mathrm{CH}_{2}\right)$, $28.91\left(\mathrm{CH}_{2}\right), 28.37\left(\mathrm{CH}_{3}\right)\left(\mathrm{NB}\right.$ masked $\left.\mathrm{CH}_{2}\right), 24.51\left(\mathrm{CH}_{2}\right)$, $24.18\left(\mathrm{CH}_{2}\right)$; HRMS MNa ${ }^{+}, \mathrm{C}_{34} \mathrm{H}_{44} \mathrm{~N}_{4} \mathrm{O}_{7}$, found 643.3098, requires 643.3108 .

$\mathrm{MeO}(L) \operatorname{Pro}(D) \operatorname{Phe}(L) \operatorname{Pro}(D) \operatorname{Phe}(L) H i v O T B S \quad(-)-17$. Colourless foam $\left(0.52 \mathrm{~g}, 71 \%\right.$ over 2 -steps); $\mathrm{R}_{f}$ (ethyl acetate) $0.78 ;[\alpha]_{\mathrm{D}}{ }^{16}-12.4\left(\mathrm{c} 1, \mathrm{CHCl}_{3}\right) ; v_{\max }$ (solution, $\mathrm{CHCl}_{3}$ ) 3416, 3290, 2957, 1743, 1658, 1515, 1451, 1254, $1052 \mathrm{~cm}^{-1} ; \delta_{\mathrm{H}}\left(400 \mathrm{MHz} ; \mathrm{CDCl}_{3}\right) 7.27-7.13(\mathrm{~m}, 10 \mathrm{H})$, $7.07(\mathrm{~d}, J=7.6 \mathrm{~Hz}, 2 \mathrm{H}), 4.87-4.82(\mathrm{~m}, 1 \mathrm{H}), 4.77-4.73$ (m, 1H), $4.41(\mathrm{dd}, J=2.4,8.4 \mathrm{~Hz}, 1 \mathrm{H}), 4.23(\mathrm{dd}, J=4.0$, $7.6 \mathrm{~Hz}, 1 \mathrm{H}), 3.96(\mathrm{~s}, 3 \mathrm{H}, 3.01-2.88(\mathrm{~m}, 4 \mathrm{H}), 2.68-2.64$ (m, 1H), $2.57-2.52(\mathrm{~m}, 1 \mathrm{H}), 2.48-2.44(\mathrm{~m}, 1 \mathrm{H}), 2.09-$ $2.02(\mathrm{~m}, 1 \mathrm{H}), 1.99-1.95(\mathrm{~m}, 1 \mathrm{H}), 1.88-1.75(\mathrm{~m}, 5 \mathrm{H})$, $1.66-1.60(\mathrm{~m}, 1 \mathrm{H}), 1.51-1.46(\mathrm{~m}, 2 \mathrm{H}), 0.92-0.90(\mathrm{~m}$, $15 \mathrm{H}), 0.40(\mathrm{~s}, 3 \mathrm{H}),-0.05(\mathrm{~s}, 3 \mathrm{H}) ; \delta_{\mathrm{C}}\left(100 \mathrm{MHz} ; \mathrm{CDCl}_{3}\right)$ 173.83 (C), 172.28 (C), 170.63(C), 170.51(C), 169.91(C), $136.72(\mathrm{C}), 135.69(\mathrm{C}), 129.44(\mathrm{CH}), 129.31(\mathrm{CH}), 128.68$ $(\mathrm{CH}), 128.34(\mathrm{CH}), 127.33(\mathrm{CH}), 126.82(\mathrm{CH}), 77.38$ $(\mathrm{CH}), 60.53(\mathrm{CH}), 58.72(\mathrm{CH}), 52.45(\mathrm{CH}), 52.28(\mathrm{CH})$, $52.09\left(\mathrm{CH}_{3}\right), 46.77\left(\mathrm{CH}_{2}\right), 46.68\left(\mathrm{CH}_{2}\right), 38.79\left(\mathrm{CH}_{2}\right)$, $38.76\left(\mathrm{CH}_{2}\right), 32.62(\mathrm{C}), 29.39\left(\mathrm{CH}_{2}\right), 28.91\left(\mathrm{CH}_{2}\right), 25.76$ $\left(\mathrm{CH}_{2}\right), 24.40\left(\mathrm{CH}_{2}\right), 23.69\left(\mathrm{CH}_{2}\right), 19.60\left(\mathrm{CH}_{3}\right), 18.07$ $\left(\mathrm{CH}_{3}\right), 16.00(\mathrm{C}),-5.00\left(\mathrm{CH}_{3}\right),-5.09\left(\mathrm{CH}_{3}\right)$; HRMS $\mathrm{MNa}^{+}$, $\mathrm{C}_{40} \mathrm{H}_{58} \mathrm{~N}_{4} \mathrm{O}_{7} \mathrm{Si}_{1}$, found 757.3955, requires 757.3972.

$\operatorname{BnO}(L) \operatorname{Hiv}(L) \operatorname{Pro}(D) \operatorname{Phe}(L) \operatorname{Pro}(D) \operatorname{PheNHBoc} \quad(-)-21$. Colourless foam $\left(0.19 \mathrm{~g}, 73 \%\right.$ over 2-steps); $\mathrm{R}_{f}$ (3:1 ethyl acetate:petroleum ether) $0.65 ;[\alpha]_{\mathrm{D}}{ }^{18}-6.4\left(\mathrm{c} 1, \mathrm{CHCl}_{3}\right)$; $v_{\max }$ (film) 3584, 3413, 2965, 2248, 1747, 1685, 1645, $1454,1172 \mathrm{~cm}^{-1} ; \delta_{\mathrm{H}}\left(400 \mathrm{MHz} ; \mathrm{CDCl}_{3}\right) 7.27-7.14(\mathrm{~m}$, $15 \mathrm{H}), 7.07-7.05(\mathrm{~m}, 1 \mathrm{H}), 5.41(\mathrm{~d}, J=7.6 \mathrm{~Hz}, 1 \mathrm{H}), 5.08$ $(\mathrm{dd}, J=12.0,12.4 \mathrm{~Hz}, 2 \mathrm{H}), 4.94(\mathrm{t}, J=4.4 \mathrm{~Hz}, 1 \mathrm{H}), 4.87$ $(\mathrm{q}, J=7.2 \mathrm{~Hz}, 1 \mathrm{H}), 4.59-4.56(\mathrm{~m}, 1 \mathrm{H}), 4.40(\mathrm{dd}, J=3.6$, $8.4 \mathrm{~Hz}, 1 \mathrm{H}), 4.32(\mathrm{dd}, J=2.8,8.4 \mathrm{~Hz}, 1 \mathrm{H}), 3.56-3.53$ $(\mathrm{m}, 2 \mathrm{H}), 2.99-2.94(\mathrm{~m}, 4 \mathrm{H}), 2.74-2.68(\mathrm{~m}, 1 \mathrm{H}), 2.61-$ $2.49(\mathrm{~m}, 2 \mathrm{H}), 2.30-2.29(\mathrm{~m}, 1 \mathrm{H}), 2.27-2.24(\mathrm{~m}, 1 \mathrm{H})$, $1.96-1.50(\mathrm{~m}, 6 \mathrm{H}), 1.39(\mathrm{~s}, 9 \mathrm{H}), 1.03(\mathrm{~d}, J=6.8 \mathrm{~Hz}, 3 \mathrm{H})$, $0.97(\mathrm{~d}, J=6.8 \mathrm{~Hz}, 3 \mathrm{H}) ; \delta_{\mathrm{C}}\left(100 \mathrm{MHz} ; \mathrm{CDCl}_{3}\right.$ ) (one carbon masked) 172.10 (C), 171.12 (C), 170.60 (C), 169.68 (C), 169.46 (C), 168.93 (C), 136.59 (C), 135.21 (C), $135.02(\mathrm{C}), 129.53(\mathrm{CH}), 129.49(\mathrm{CH}), 128.63(\mathrm{CH})$, $128.57(\mathrm{CH}), 128.45(\mathrm{CH}), 128.39(\mathrm{CH}), 128.32(\mathrm{CH})$, $126.93(\mathrm{CH}), 126.84(\mathrm{CH}), 79.72(\mathrm{CH}), 77.58(\mathrm{CH}), 67.18$ $\left(\mathrm{CH}_{2}\right), 66.93\left(\mathrm{CH}_{2}\right), 60.36(\mathrm{CH}), 58.42(\mathrm{CH}), 53.86(\mathrm{CH})$, $52.44(\mathrm{CH}), 46.88\left(\mathrm{CH}_{2}\right), 46.73\left(\mathrm{CH}_{2}\right), 38.97\left(\mathrm{CH}_{2}\right), 31.92$ $\left(\mathrm{CH}_{2}\right), 30.18\left(\mathrm{CH}_{3}\right), 29.69\left(\mathrm{CH}_{2}\right), 28.38\left(\mathrm{CH}_{2}\right), 24.23$ $\left(\mathrm{CH}_{2}\right), \quad 18.62\left(\mathrm{CH}_{3}\right), \quad 17.15 \quad\left(\mathrm{CH}_{3}\right)$; HRMS $\mathrm{MNa}^{+}$, $\mathrm{C}_{45} \mathrm{H}_{56} \mathrm{~N}_{4} \mathrm{O}_{9}, 819.3899$, requires 819.3945.

(20) Alternaramide (-)-1. Colourless crystalline solid (77 mg, $48 \%$ over 3 -steps) m.p. $217-218.5{ }^{\circ} \mathrm{C}$ (recrystallised from $\mathrm{CH}_{2} \mathrm{Cl}_{2}$ / petroleum ether); $\mathrm{R}_{f}$ (3:1 ethyl 
acetate:petroleum ether) $0.48 ;[\alpha]_{\mathrm{D}}^{19}-2.8(\mathrm{c} 0.5, \mathrm{MeOH})$; $v_{\max }$ (film) 3584, 3480, 3300, 3029, 2961, 2929, 2875, 2248, 1744, 1683, 1643, 1634, 1529, 1452, 1275, 1094 $\mathrm{cm}^{-1} ; \delta_{\mathrm{H}}\left(400 \mathrm{MHz} ; \mathrm{CDCl}_{3}\right) 8.12(\mathrm{~d}, J=9.6 \mathrm{~Hz}, 1 \mathrm{H}), 7.32$ $-7.17(\mathrm{~m}, 11 \mathrm{H}), 5.34(\mathrm{~d}, J=2.4 \mathrm{~Hz}, 1 \mathrm{H}), 5.02-4.91(\mathrm{~m}$, $2 \mathrm{H}), 4.85(\mathrm{~d}, J=7.2 \mathrm{~Hz}, 1 \mathrm{H}), 4.30(\mathrm{dd}, J=5.2,8.8 \mathrm{~Hz}$, $1 \mathrm{H}), 3.46-3.41(\mathrm{~m}, 1 \mathrm{H}), 3.35(\mathrm{dd}, J=9.2,13.2 \mathrm{~Hz}, 1 \mathrm{H})$, $3.26(\mathrm{~m}, 2 \mathrm{H}), 3.03-2.99(\mathrm{~m}, 2 \mathrm{H}), 2.90(\mathrm{dd}, J=5.2,13.2$ $\mathrm{Hz}, 1 \mathrm{H}), 2.54-2.49(\mathrm{~m}, 2 \mathrm{H}), 2.44-2.42(\mathrm{~m}, 1 \mathrm{H}), 1.99-$ $1.93(\mathrm{~m}, 2 \mathrm{H}), 1.93-1.84(\mathrm{~m}, 2 \mathrm{H}), 1.78-1.72(\mathrm{~m}, 1 \mathrm{H})$, $1.69-1.63(\mathrm{~m}, 1 \mathrm{H}), 1.46$ (sept, $J=6.8 \mathrm{~Hz}, 1 \mathrm{H}), 0.82(\mathrm{~d}, J$ $=6.8 \mathrm{~Hz}, 3 \mathrm{H}), 0.71(\mathrm{~d}, J=6.8 \mathrm{~Hz}, 3 \mathrm{H}) ; \delta_{\mathrm{C}}(100 \mathrm{MHz}$; $\left.\mathrm{CDCl}_{3}\right) 171.16(\mathrm{C}), 169.66(\mathrm{C}), 169.19(\mathrm{C}), 168.86(\mathrm{C})$, $167.26(\mathrm{C}), 136.49(\mathrm{C}), 135.00(\mathrm{C}), 128.66(\mathrm{CH}), 128.30$ $(\mathrm{CH}), 127.58(\mathrm{CH}), 127.36(\mathrm{CH}), 126.29(\mathrm{C}), 125.48(\mathrm{C})$, $75.95(\mathrm{CH}), 58.92(\mathrm{CH}), 57.28(\mathrm{CH}), 51.85(\mathrm{CH}), 51.13$ $(\mathrm{CH}), 45.89\left(\mathrm{CH}_{2}\right), 44.94\left(\mathrm{CH}_{2}\right), 39.43\left(\mathrm{CH}_{2}\right), 35.95$ $\left(\mathrm{CH}_{2}\right), 28.65(\mathrm{CH}), 28.50\left(\mathrm{CH}_{2}\right), 23.75\left(\mathrm{CH}_{2}\right), 23.48$ $\left(\mathrm{CH}_{2}\right), 18.09\left(\mathrm{CH}_{3}\right), 14.83\left(\mathrm{CH}_{3}\right)$; HRMS $\mathrm{MNa}^{+}$, $\mathrm{C}_{33} \mathrm{H}_{40} \mathrm{~N}_{4} \mathrm{O}_{6}$, found 611.2833, requires 611.2846.

(21) See supporting information for copies of the ${ }^{1} \mathrm{H},{ }^{13} \mathrm{C}$ NMR spectra for synthetically prepared alternaramide (-)-1.

(22) Crystal data for (-)-1: $\quad \mathrm{C}_{33} \mathrm{H}_{40} \mathrm{~N}_{4} \mathrm{O}_{6}, \quad \mathrm{M}=588.69$, orthorhombic, $P 2{ }_{1} 2_{1}{ }_{1} ; a=12.0808(4) b=22.0624(7), c=$ $34.9650(11) \AA, V=9319.3(5) \AA^{3} ; D_{\text {calc }}=1.259 \mathrm{~g} / \mathrm{cm}^{3}$; $\mu \square(\mathrm{Mo}-\mathrm{K} \alpha)=0.087 \mathrm{~mm}^{-1} ; \lambda=0.71073 \AA, \mathrm{T}=150(2) \mathrm{K} ;$ 104556 total reflections, 26198 unique data $\left(R_{\mathrm{int}}=\right.$ 0.0456); Solved by direct methods and refined on $F^{2}$ values to give $R 1=0.0473\left[F^{2}>2 \sigma\left(F^{2}\right)\right]$ (19948 observed reflections), $w R 2=0.1107$ (all data). Absolute structure parameter $=-0.09(22)^{23,24}$. CCDC: 801185 contains the supplementary crystallographic data for compound (-)-1. These data can be obtained free of charge via http://www.ccdc.cam.ac.uk/data_request/cif or from the Cambridge Crystallographic Data Centre, 12 Union Road, Cambridge, CB2 1EZ, UK; fax: (+44) 1223336033 or email: deposit@ccdc.cam.ac.uk..

(23) Flack, H. D., Acta Crystallogr., 1983, A39, 876.

(24) Parsons, S., University of Edinburgh. 
Alexandra E. Horton, Oliver S. May, Mark R. J. Elsegood and Marc C. Kimber* The total synthesis of the marine-derived cyclic depsipeptide alternaramide

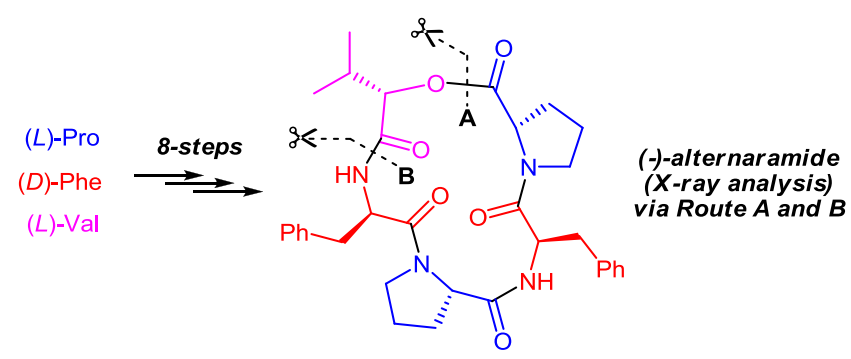

